\title{
Swallowing dysfunction as risk factor for undernutrition in older people admitted to Swedish short-term care: a cross-sectional study
}

\author{
Patricia Hägglund ${ }^{1}$ (D) $\cdot$ Anna Fält ${ }^{2} \cdot$ Mary Hägg $^{3,4} \cdot$ Per Wester $^{5,6} \cdot$ Eva Levring Jäghagen ${ }^{1}$
}

Received: 21 December 2017 / Accepted: 3 April 2018 / Published online: 16 April 2018

(c) The Author(s) 2018

\begin{abstract}
Background Swallowing dysfunction and risk of undernutrition increase the risk of pneumonia, morbidity, and mortality. Short-term care is an unexplored care context, where many older people stay yearly.

Aim This cross-sectional study aimed to describe and analyze the relationship between swallowing dysfunction and risk of undernutrition among older people in short-term care, including potential gender-related differences.

Methods In total, 391 people (209 women), aged $\geq 65$ years (median age 84 years) and admitted to short-term care in five Swedish counties participated. They went through a timed water swallow test to assess swallowing dysfunction, including abnormal swallowing capacity and signs of aspiration (i.e., cough and voice change). Risk for undernutrition was assessed using the Minimal Eating Observation and Nutrition Form-version II.

Results Swallowing dysfunction was observed in 248 of 385 (63\%) participants, including abnormal swallowing capacity in 213 of 385 (55\%) and aspiration signs in 127 of 377 (34\%). Abnormal swallowing capacity was more frequent among women $(p=0.030)$, whereas men with normal swallowing capacity exhibited signs of aspiration more frequently ( $\operatorname{cough} p=0.038$, voice change $p=0.004)$. Risk of undernutrition was found in 91 of $390(23 \%)$ participants, more frequently among women $(p=0.007)$. A logistic regression model revealed an increased risk of undernutrition among older people with abnormal swallowing capacity (OR 1.74, 95\% CI 1.04-2.92, $p=0.034$ ).

Conclusions The high prevalence of swallowing dysfunction and risk of undernutrition highlight the need for a systematic screening program and feasible treatment to improve swallowing function for adequate and safe food intake among older people in short-term care.
\end{abstract}

Clinical trial registration This study was registered with ClinicalTrials.gov on July 4, 2016, under NCT02825927.

Electronic supplementary material The online version of this article (https://doi.org/10.1007/s40520-018-0944-7) contains supplementary material, which is available to authorized users.

Patricia Hägglund

patricia.hagglund@umu.se

1 Oral and Maxillofacial Radiology, Department of Odontology, Faculty of Medicine, Umeå University, Umeå, Sweden

2 Clinical Epidemiology and Biostatistics, School of Medical Sciences, Örebro University, Örebro, Sweden

3 Speech and Swallowing Centre, Department of Otorhinolaryngology, Hudiksvall Hospital, County Council of Gävleborg, Hudiksvall, Sweden

4 Centre for Research and Development, Uppsala University, Uppsala, Sweden

5 Department of Public Health and Clinical Medicine, Umeå University, Umeå, Sweden

6 Department of Clinical Science, Danderyd Hospital, Karolinska Institute, Stockholm, Sweden 
Keywords Swallowing disorders $\cdot$ Aspiration $\cdot$ Malnutrition $\cdot$ Gender $\cdot$ Dysphagia $\cdot$ Elderly care

\section{Introduction}

Dysphagia and malnutrition are prevalent among older people and have a negative impact on health, with poor recovery and an increased risk of pneumonia, morbidity, and mortality [1-4]. However, dysphagia and malnutrition are often unrecognized and poorly treated among older people $[2,4]$.

The term dysphagia refers to subjectively experienced symptoms of swallowing dysfunction, and is often used to describe objectively observed or measured swallowing dysfunction. Common signs of dysfunction are reduced swallowing capacity and aspiration signs, such as cough and wet or gurgly voice [5-7]. The reported prevalence of dysphagia among older people varies from 11 to $80 \%$; the wide range is explained by differences in study groups and selection, screening or assessment tools, and the definition of dysphagia [3]. Dysphagia risk is associated with neurological diseases, functional capacity, polymedication, and multimorbidity, and increases with age and frailty $[8,9]$. Dysphagia has been found to be a risk factor for malnutrition in hospitalized older people [9].

Malnutrition is defined as any nutritional imbalance. In acute, chronic, or transitional care settings, the detection and management of adult undernutrition is a primary concern, as undernutrition is associated with poorer health and increased mortality $[2,10]$. A varying prevalence of malnutrition has been reported for different settings: $50 \%$ in rehabilitation, $38.7 \%$ in general hospital, $13.8 \%$ in nursing homes, and 5.8\% in community [11]. The recommended definition of malnutrition is based on at least two of six characteristics: insufficient energy intake, weight loss, loss of muscle mass, loss of subcutaneous fat, localized or generalized fluid accumulation, and diminished functional status [2].

Short-term care units provide the basic nursing care for days to months for older people recovering after being discharged from the hospital, waiting for homecare placement, undergoing rehabilitation, as recurrent relief for family members who are informal caregivers (i.e., respite care), or end-of-life care [12]. Approximately 8000 older people with different diagnoses are admitted to Swedish short-term care units annually, but little is known regarding the frequency of dysphagia and poor nutritional status among them. Therefore, the aims of the present study were to describe the prevalence of swallowing dysfunction, including swallowing capacity and aspiration signs, and the risk of undernutrition. We also analyzed the relationship between swallowing dysfunction and the risk of undernutrition among older people with different diagnoses in Swedish short-term care and potential gender-related differences.
Fig. 1 Flow chart of enrollment and assessments

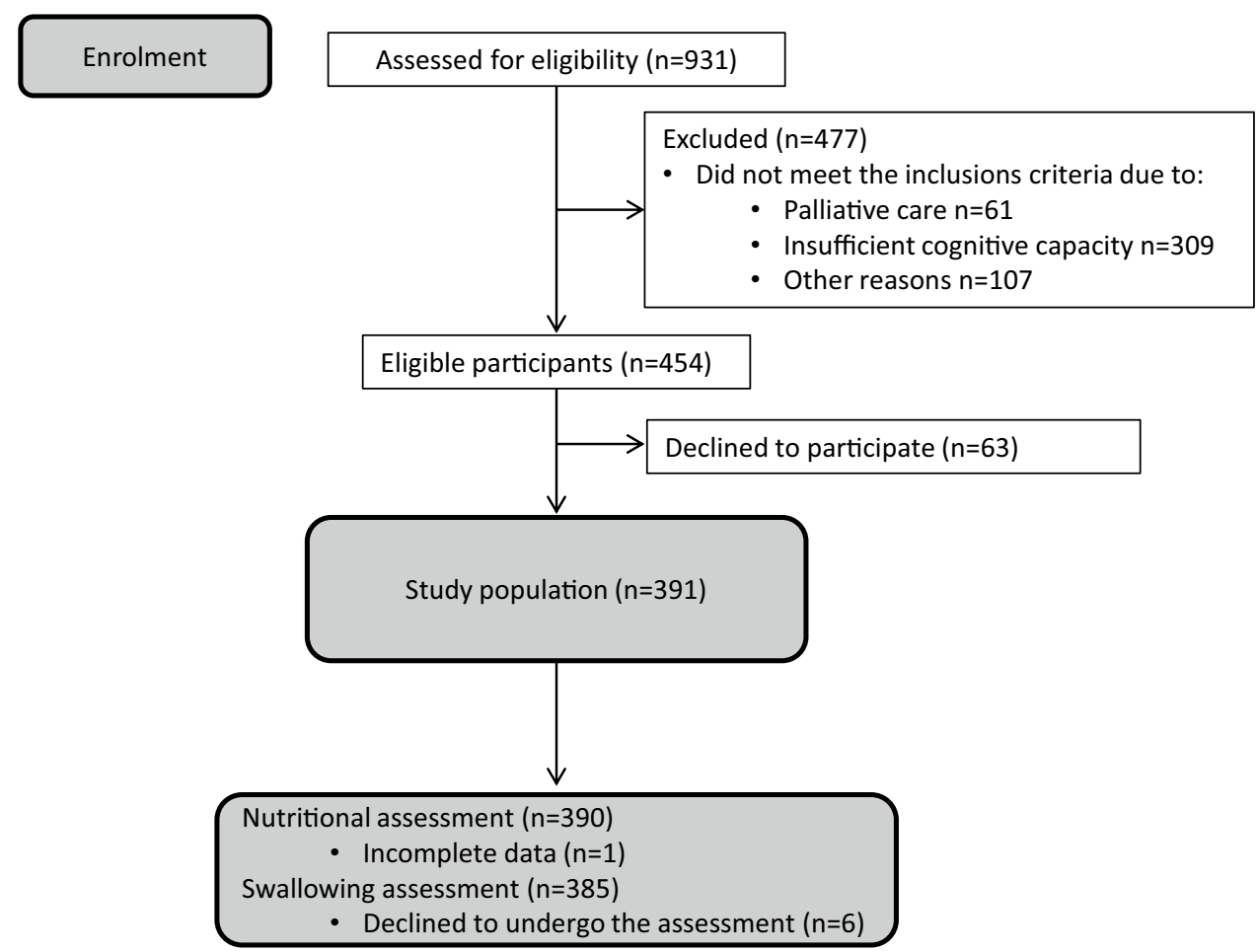


Table 1 Participant characteristics and distribution of results between gender

\begin{tabular}{|c|c|c|c|c|}
\hline & $\begin{array}{l}\text { Total } \\
n=391\end{array}$ & $\begin{array}{l}\text { Male } \\
n=182\end{array}$ & $\begin{array}{l}\text { Female } \\
n=209\end{array}$ & $P$ value \\
\hline Median age (IQR) & $84(78-89)$ & $81(76-88)$ & $85(80-90)$ & $0.001^{\mathrm{a}}$ \\
\hline Age, years & & & & $0.004^{\mathrm{b}}$ \\
\hline$<75$ & $63(16.1)$ & $39(21.4)$ & $24(11.5)$ & \\
\hline $75-84$ & $149(38.1)$ & $74(40.7)$ & $75(35.9)$ & \\
\hline$\geq 85$ & $179(45.8)$ & $69(37.9)$ & $110(52.6)$ & \\
\hline Multimorbidity* & & & & $0.982^{b}$ \\
\hline Yes & $206(52.7)$ & $96(52.7)$ & $110(52.6)$ & \\
\hline No & $185(47.3)$ & $86(47.3)$ & $99(47.4)$ & \\
\hline Dysphagia risk condition** $(n=389)$ & & & & $<0.001^{\mathrm{b}}$ \\
\hline Yes & $154(39.6)$ & $96(53.0)$ & $58(27.9)$ & \\
\hline No & $235(60.4)$ & $85(47.0)$ & $125(72.1)$ & \\
\hline \multicolumn{5}{|l|}{ Diagnosis causing short-term care $^{\dagger}$} \\
\hline Stroke & $87(22.3)$ & $52(28.6)$ & $35(16.7)$ & \\
\hline Muscle skeletal disease & $85(21.7)$ & $22(12.1)$ & $63(30.1)$ & \\
\hline Mild cognitive impairment & $47(12.0)$ & $20(11.0)$ & $27(12.9)$ & \\
\hline Cardiovascular & $42(10.7)$ & $20(11.0)$ & $22(10.5)$ & \\
\hline Other neurological & $33(8.4)$ & $25(13.7)$ & $8(3.8)$ & \\
\hline Cancer & $24(6.1)$ & $11(6.0)$ & $13(6.2)$ & \\
\hline Diabetes & $16(4.1)$ & $6(3.3)$ & $10(4.8)$ & \\
\hline Respiratory & $14(3.6)$ & $7(3.8)$ & $7(3.3)$ & \\
\hline Psychiatric & $11(2.8)$ & $0(0.0)$ & $11(5.3)$ & \\
\hline Gastrointestinal & $9(2.3)$ & $4(2.2)$ & $5(2.4)$ & \\
\hline Diverse & $9(2.3)$ & $7(3.8)$ & $2(1.0)$ & \\
\hline Kidney & $7(1.8)$ & $2(1.1)$ & $5(2.4)$ & \\
\hline Poor general health & $5(1.3)$ & $5(2.7)$ & $0(0.0)$ & \\
\hline Unknown & $2(0.5)$ & $1(0.5)$ & $1(0.5)$ & \\
\hline Katz-ADL $(n=385)$ & & & & $0.005^{\mathrm{b}}$ \\
\hline A & $28(7.3)$ & $14(7.8)$ & $14(6.8)$ & \\
\hline B-D & $165(42.8)$ & $61(34.1)$ & $104(50.5)$ & \\
\hline $\mathrm{E}-\mathrm{G}$ & $192(49.9)$ & $104(58.1)$ & $88(42.7)$ & \\
\hline Swallowing dysfunction*** $(n=385)$ & & & & $0.991^{b}$ \\
\hline Yes & $248(63.4)$ & $116(64.4)$ & $132(64.4)$ & \\
\hline No & $137(35.0)$ & $64(35.6)$ & $73(35.6)$ & \\
\hline Swallowing capacity $(n=385)$ & & & & $0.004^{\mathrm{a}}$ \\
\hline Median mL/s (IQR) & $8.5(4.2-12.0)$ & $10.0(5.3-13.5)$ & $7.1(3.7-11.4)$ & \\
\hline Capacity $(n=385)$ & & & & $0.030^{\mathrm{b}}$ \\
\hline$<10 \mathrm{~mL} / \mathrm{s}$ & $213(54.5)$ & $89(49.4)$ & $124(60.5)$ & \\
\hline$\geq 10 \mathrm{~mL} / \mathrm{s}$ & $172(44.0)$ & $91(50.6)$ & $81(39.5)$ & \\
\hline Signs of aspiration $* * * *(n=377)$ & & & & $0.034^{\mathrm{b}}$ \\
\hline Yes & $127(33.7)$ & $70(39.1)$ & $57(28.8)$ & \\
\hline No & $250(66.3)$ & $109(60.9)$ & $141(71.2)$ & \\
\hline Cough $(n=377)$ & & & & $0.045^{\mathrm{b}}$ \\
\hline Yes & $90(23.9)$ & $51(28.5)$ & 39 (19.7) & \\
\hline No & $287(76.1)$ & $128(71.5)$ & $159(80.3)$ & \\
\hline Voice change $(n=368)$ & & & & $0.034^{\mathrm{b}}$ \\
\hline Yes & $65(17.7)$ & $39(22.0)$ & $26(13.6)$ & \\
\hline No & $303(82.3)$ & $138(78.0)$ & $165(86.4)$ & \\
\hline Voice change without cough $(n=368)$ & & & & $0.676^{\mathrm{b}}$ \\
\hline Yes & $37(10.1)$ & $19(10.7)$ & $18(9.4)$ & \\
\hline No & $331(89.9)$ & $158(89.3)$ & $173(90.6)$ & \\
\hline
\end{tabular}


Table 1 (continued)

\begin{tabular}{llllr}
\hline & $\begin{array}{l}\text { Total } \\
n=391\end{array}$ & $\begin{array}{l}\text { Male } \\
n=182\end{array}$ & \multicolumn{1}{l}{$\begin{array}{l}\text { Female } \\
n=209\end{array}$} & $P$ value \\
\hline Risk of undernutrition $(n=390)$ & & & & $0.007^{\mathrm{b}}$ \\
Yes & $91(23.3)$ & $31(17.1)$ & $60(28.7)$ & \\
No & $299(76.7)$ & $150(82.9)$ & $149(71.3)$ & \\
\hline
\end{tabular}

Data are given as $n(\%)$ or median (interquartile range)

*Three or more diagnoses from minimum three different organs/organ systems

**Any condition that may contribute to dysphagia, i.e., neurological disease, stroke, traumatic brain injury, and chronic obstructive pulmonary disease

***Abnormal swallowing capacity and/or signs of aspiration

****Signs of aspiration were defined as cough, voice change, and/or voice change without cough

${ }^{\dagger}$ The main diagnosis causing short-term care, e.g., a person with previous stroke who recently broke a hip has the main diagnosis muscle skeletal disease

${ }^{a}$ Mann-Whitney $U$ test

${ }^{\mathrm{b}}$ Chi-squared test

\section{Subjects and methods}

\section{Subjects}

The study population was included in a multidisciplinary and multicenter project including short-term care residents in five counties in Sweden [13]. They were $\geq 65$ years old, admitted for at least 3 days, able to understand Swedish, had the cognitive capacity sufficient to give informed consent, and the ability to participate in the clinical measurements. The latter was based on medical records and judged by the responsible nurse at the short-term care unit. Older people who received end-of-life care were excluded. Of 931 available residents in the short-term care units, 477 did not fulfill the inclusion criteria and 63 declined to participate. A total of 391 older people were finally included in the study. (Fig. 1).

\section{Data collection}

The clinical assessments were conducted in the short-term care unit by formally trained professionals (eight registered dental hygienists and one speech-language pathologist) who had received specific training on performing all assessments before the study started. Clinical data regarding participant age, gender, height, weight, and medical diagnosis based on social service and nursing documentation, and cause of stay were collected (Table 1). The participants were considered multimorbid if suffering from $\geq 3$ diagnoses from a minimum of three different organ/organ systems.

Functional status was assessed using the modified Katz Index of Activities of Daily Living (Katz-ADL) recommended for short-term care [14, 15] and was judged by the responsible nurse. The scores were yes or no for independence in six functions: bathing, dressing, toileting, transferring, continence, and feeding. The Katz-ADL ranges from $\mathrm{A}-\mathrm{G}, \mathrm{A}$ indicates independence (score yes in all six functions), B-D indicates moderate dependency, and $\mathrm{E}-\mathrm{G}$ indicates total dependency $[14,16]$.

\section{Primary outcome/measurements}

A timed water swallow test (WST) of swallowing capacity was used to assess swallowing dysfunction by measuring swallowing capacity and clinical signs of aspiration (i.e., cough and voice change) [5]. Swallowing capacity has been shown to be a sensitive indicator for identifying patients at risk of swallowing dysfunction. Cough and voice change after swallowing is an indicator of water penetrating the larynx or, worse, resulting in aspiration into trachea [6]. Before proceeding with the WST, a teaspoon test was carried out to identify subjects with major risk of aspiration. The participant swallowed three teaspoons of water; if aspiration signs were observed, the WST was not carried out and a WST score of $0 \mathrm{~mL} / \mathrm{s}$ recorded. For the timed WST, the participant was instructed to drink $150 \mathrm{~mL}$ of water from a glass as rapidly but safely as possible, and to stop if difficulties arose. The participants were requested to use their normal swallowing behavior, including any swallowing compensatory strategy, e.g., postural adjustment, that they used in daily life. Aspiration signs during and after swallowing were recorded, and the residual volume was measured if the WST was interrupted or incomplete. The swallowing capacity was calculated as the milliliters of swallowed water divided by time. In accordance with the previous studies, swallowing capacity $<10 \mathrm{~mL} / \mathrm{s}$ was considered abnormal $[5,6]$.

The Minimal Eating Observation and Nutrition Formversion II (MEONF-II) was conducted by a trained nurse responsible for the participant. It was used to assess the risk 
of undernutrition based on unintentional weight loss, low body mass index/short calf circumference, eating difficulties (food intake, chewing/swallowing, decreased energy/appetite), and clinical signs of undernutrition [17]. The MEONFII score ranges from 0 to 8 ; a score of $0-2$ indicates no or low risk of undernutrition, 3-4 moderate risk of undernutrition, and $\geq 5$ high risk of undernutrition. The cutoff for risk of undernutrition was defined as a MEONF-II score $\geq 3$.

\section{Statistical analysis}

Statistical analyses were performed using IBM/SPSS Statistics 23. Categorical variables were analyzed by the Chisquared test or Fisher exact test. For continuous variables, the Mann-Whitney $U$ or Kruskal-Wallis test was used. Logistic regression was used to calculate the odds ratio (OR) with 95\% confidence interval (CI) for the association between swallowing capacity and risk of undernutrition. Adjustments were made for possible confounding variables. $p<0.05$ was considered significant.

\section{Results}

\section{Subjects}

Of the 391 older people included in this study, 209 were women (median age 85 years, range 65-100 years) and 182 men (median age 81 years, range 65-98 years) (Table 1). Swallowing assessment was carried out in 385 subjects (missing $n=6$ ) and nutritional assessment in 390 subjects (missing $n=1$ ) (Fig. 1). The causes of admission for shortterm care were as follows: $76(19 \%)$ respite care, $70(18 \%)$ acute short-term care, $58(15 \%)$ recovery after hospitalization, $50(13 \%)$ rehabilitation, $33(8 \%)$ waiting for care placement, 26 (7\%) further assessment, and 78 (20\%) unknown reason.

\section{Swallowing dysfunction}

A total of 248 of the 385 (63\%) participants exhibited swallowing dysfunction with either abnormal swallowing capacity and/or signs of aspiration (Table 1). Swallowing dysfunction was associated with decreased functional status (moderate or total dependency according to Katz-ADL; $p=0.015)$ and increased age ( $75-84$ years or $\geq 85$ years, $p=0.027$; Table 2). However, no significant association was found between swallowing dysfunction and gender $(p=0.991)$, health conditions with increased risk of suffering from dysphagia (i.e., health conditions that can contribute to swallowing dysfunction; $p=0.429$ ), multimorbidity $(p=0.444)$, or mild cognitive impairment $(p=0.287)$.
Table 2 Comparison of participants with normal swallowing function and those with swallowing dysfunction

\begin{tabular}{|c|c|c|c|}
\hline & $\begin{array}{l}\text { Normal } \\
\text { swallowing } \\
n=137\end{array}$ & $\begin{array}{l}\text { Swallowing } \\
\text { dysfunction } \dagger \\
n=248\end{array}$ & $p$ value \\
\hline Gender & & & 0.991 \\
\hline Male & 64 (46.7) & $116(46.8)$ & \\
\hline Female & 73 (53.3) & $132(53.2)$ & \\
\hline Age, years & & & 0.027 \\
\hline$<75$ & 30 (21.9) & 32 (12.9) & \\
\hline $75-84$ & $55(40.1)$ & $93(37.5)$ & \\
\hline$\geq 85$ & $52(38.0)$ & $123(49.6)$ & \\
\hline Multimorbidity* & & & 0.444 \\
\hline Yes & $69(50.4)$ & $135(54.4)$ & \\
\hline No & 68 (49.6) & $113(45.6)$ & \\
\hline $\begin{array}{l}\text { Dysphagia risk condi- } \\
\text { tion** }\end{array}$ & & & 0.429 \\
\hline Yes & $50(36.8)$ & $101(40.9)$ & \\
\hline No & $86(63.2)$ & $146(59.1)$ & \\
\hline $\begin{array}{l}\text { Mild cognitive impair- } \\
\text { ment*** }\end{array}$ & & & 0.287 \\
\hline Yes & 19 (22.4) & $24(16.7)$ & \\
\hline No & 66 (77.6) & $120(83.3)$ & \\
\hline Katz-ADL & & & 0.015 \\
\hline A & $14(10.5)$ & $14(5.7)$ & \\
\hline B-D & 66 (49.6) & $98(39.8)$ & \\
\hline $\mathrm{E}-\mathrm{G}$ & $53(39.8)$ & $134(54.5)$ & \\
\hline
\end{tabular}

Data are presented as $n(\%)$. Statistical analysis was performed using the Chi-squared test

Defined as abnormal swallowing capacity $(<10 \mathrm{~mL} / \mathrm{s})$ and/or signs of aspiration

*Defined as three or more diagnoses from three different organs/ organ systems

**Any condition that may contribute to dysphagia, see Table 1

***Medical record of mild dementia without any dysphagia risk condition

\section{Abnormal swallowing capacity}

Abnormal swallowing capacity $(<10 \mathrm{~mL} / \mathrm{s})$ was found in 213/385 (55\%) participants (Table 1), including 11 with an unsafe teaspoon test and registered swallowing capacity $0 \mathrm{~mL} / \mathrm{s}$. The prevalence of swallowing capacity $<10 \mathrm{~mL} / \mathrm{s}$ was significantly higher among women $(124 / 205,60 \%)$ than men $(89 / 180,49 \% ; p=0.030)$. Swallowing capacity $<10 \mathrm{~mL} / \mathrm{s}$ was associated with signs of aspiration $(p<0.001$; Table 3$)$. However, no association was found between abnormal swallowing capacity and age $(p=0.123)$, Katz-ADL $(p=0.073)$, multimorbidity $(p=0.291)$, dysphagia risk conditions $(p=0.472)$, or mild cognitive impairment $(p=0.073)$. 
Table 3 Comparisons between participants based on swallowing capacity and risk of undernutrition

\begin{tabular}{|c|c|c|c|c|c|c|}
\hline & $\begin{array}{l}\text { Capacity } \geq 10 \mathrm{~mL} / \mathrm{s} \\
n=172\end{array}$ & $\begin{array}{l}\text { Capacity }<10 \mathrm{~mL} / \mathrm{s} \\
n=213\end{array}$ & $p$ value & $\begin{array}{l}\text { No risk undernutrition } \\
n=299\end{array}$ & $\begin{array}{l}\text { Risk undernutrition } \\
n=91\end{array}$ & $p$ value \\
\hline Gender & & & 0.030 & & & 0.007 \\
\hline Male & $91(52.9)$ & $89(41.8)$ & & $150(50.2)$ & $31(34.1)$ & \\
\hline Female & $81(47.1)$ & $124(58.2)$ & & $149(49.8)$ & $60(65.9)$ & \\
\hline Age, years & & & 0.123 & & & 0.991 \\
\hline$<75$ & $33(19.2)$ & $29(13.6)$ & & $48(16.1)$ & $15(16.5)$ & \\
\hline $75-84$ & $70(40.7)$ & $78(36.6)$ & & $114(38.1)$ & $35(38.5)$ & \\
\hline$\geq 85$ & $69(40.1)$ & $106(49.8)$ & & $137(45.8)$ & $41(45.0)$ & \\
\hline Multimorbidity* & & & 0.291 & & & 0.215 \\
\hline Yes & $86(50.0)$ & $118(55.4)$ & & $152(50.8)$ & $53(58.2)$ & \\
\hline No & $86(50.0)$ & $95(44.6)$ & & $147(49.2)$ & $38(41.8)$ & \\
\hline Dysphagia risk condition** & & & 0.472 & & & 0.945 \\
\hline Yes & $64(37.4)$ & $87(41.0)$ & & $118(39.6)$ & $36(40.0)$ & \\
\hline No & $107(62.6)$ & $125(59.0)$ & & $180(60.4)$ & $54(60.0)$ & \\
\hline Mild cognitive impairment $* * *$ & & & 0.073 & & & 0.278 \\
\hline Yes & $25(23.8)$ & $18(14.5)$ & & $36(20.1)$ & $45(86.5)$ & \\
\hline No & $80(76.2)$ & $106(85.5)$ & & $143(79.9)$ & $7(13.5)$ & \\
\hline Katz-ADL & & & 0.073 & & & 0.607 \\
\hline A & $15(8.9)$ & $13(6.2)$ & & $23(7.8)$ & $5(5.5)$ & \\
\hline B-D & $81(48.2)$ & $83(39.3)$ & & $127(43.4)$ & $37(40.7)$ & \\
\hline $\mathrm{E}-\mathrm{G}$ & $72(42.9)$ & $115(54.5)$ & & $143(48.8)$ & $49(53.8)$ & \\
\hline Signs of aspiration $* * * *$ & & & $<0.001$ & & & 0.083 \\
\hline Yes & $35(20.6)$ & $92(44.4)$ & & $92(31.5)$ & $35(41.7)$ & \\
\hline No & $135(79.4)$ & $115(55.6)$ & & $200(68.5)$ & $49(58.3)$ & \\
\hline Cough & & & 0.002 & & & 0.156 \\
\hline Yes & $28(16.5)$ & $62(30.0)$ & & $65(22.3)$ & $25(70.2)$ & \\
\hline No & $142(83.5)$ & $145(70.0)$ & & $227(77.7)$ & $59(29.8)$ & \\
\hline Voice change & & & $<0.001$ & & & 0.027 \\
\hline Yes & $9(5.3)$ & $56(28.3)$ & & $45(15.5)$ & $20(26.3)$ & \\
\hline No & $161(94.7)$ & $142(71.7)$ & & $246(84.5)$ & $56(73.7)$ & \\
\hline Voice change without cough & & & $<0.001$ & & & 0.317 \\
\hline Yes & $7(4.1)$ & $30(14.1)$ & & $27(9.3)$ & $10(13.2)$ & \\
\hline No & $163(94.8)$ & $168(78.9)$ & & $264(90.7)$ & $66(86.8)$ & \\
\hline Swallowing dysfunction***** & & & & & & 0.033 \\
\hline Yes & NA & NA & & $182(61.5)$ & $65(73.9)$ & \\
\hline No & NA & NA & & $114(38.5)$ & $23(26.1)$ & \\
\hline
\end{tabular}

Data are presented as $n(\%)$. Statistical analysis was performed using the Chi-squared test

NA not applicable

*Three or more diagnoses from minimum three different organs/organ systems

**Any condition that may contribute to dysphagia, see Table 1

***Medical record of mild dementia without any dysphagia risk condition

*****Meaning cough, voice change, and/or voice change without cough

*****Abnormal swallowing capacity and/or signs of aspiration

\section{Signs of aspiration}

Aspiration signs were found in 127/377 (34\%) participants; cough appeared in $90(24 \%)$, voice change in $65(18 \%)$, and voice change without cough in $37(10 \%)$ participants
(Table 1). Eighty-five of 120 (71\%) participants with aspiration signs also had abnormal swallowing capacity (Table 3). When analyzing all subjects independent of swallowing capacity, aspiration signs during the WST were significantly more common among men than women 
Table 4 Association between abnormal swallowing capacity and risk of undernutrition $(n=378)$

\begin{tabular}{|c|c|c|c|c|c|c|}
\hline & $\begin{array}{l}\text { No risk undernutri- } \\
\text { tion } n(\%)\end{array}$ & $\begin{array}{l}\text { Risk undernutrition } \\
n(\%)\end{array}$ & $\begin{array}{l}\text { Model } 1 \\
\text { OR }(95 \% \text { CI })\end{array}$ & $p$ value & $\begin{array}{l}\text { Model } 2 \\
\text { OR }(95 \% \text { CI })\end{array}$ & $p$ value \\
\hline \multicolumn{7}{|l|}{ Capacity } \\
\hline$\geq 10 \mathrm{~mL} / \mathrm{s}$ & $139(82.7)$ & $29(17.3)$ & 1.00 (ref) & & 1.00 (ref) & \\
\hline$<10 \mathrm{~mL} / \mathrm{s}$ & $151(71.9)$ & $59(28.1)$ & $1.90(1.15-3.13)$ & 0.012 & $1.74(1.04-2.92)$ & 0.034 \\
\hline \multicolumn{7}{|l|}{ Gender } \\
\hline Male & $146(83.0)$ & $30(17.0)$ & 1.00 (ref) & & 1.00 (ref) & \\
\hline Female & $144(71.3)$ & $58(28.7)$ & $1.95(1.20-3.18)$ & 0.008 & $1.93(1.18-3.25)$ & 0.010 \\
\hline \multicolumn{7}{|l|}{ Age, years } \\
\hline$<75$ & $45(76.3)$ & $14(23.7)$ & 1.00 (ref) & & 1.00 (ref) & \\
\hline $75-84$ & $111(76.0)$ & $35(24.0)$ & $0.98(0.49-1.96)$ & 0.960 & $0.90(0.43-1.87)$ & 0.783 \\
\hline$\geq 85$ & $134(77.5)$ & $39(22.5)$ & $0.96(0.49-1.88)$ & 0.900 & $0.73(0.35-1.54)$ & 0.418 \\
\hline \multicolumn{7}{|l|}{ Katz-ADL } \\
\hline A & $23(82.1)$ & $5(17.9)$ & 1.00 (ref) & & 1.00 (ref) & \\
\hline B-D & $126(77.3)$ & $37(22.7)$ & $1.34(0.48-3.77)$ & 0.579 & $1.30(0.45-3.80)$ & 0.627 \\
\hline $\mathrm{E}-\mathrm{G}$ & $141(75.4)$ & $46(24.6)$ & $1.58(0.57-4.37)$ & 0.382 & $1.49(0.52-4.27)$ & 0.456 \\
\hline \multicolumn{7}{|c|}{ Multimorbidity* } \\
\hline No & $141(79.2)$ & $37(20.8)$ & 1.00 (ref) & & 1.00 (ref) & \\
\hline Yes & $149(74.5)$ & $51(25.5)$ & $1.35(0.84-2.17)$ & 0.216 & $1.25(0.77-2.05)$ & 0.369 \\
\hline
\end{tabular}

Model 1, unadjusted

Model 2, adjusted for gender, age, Katz-ADL, multimorbidity and based on 378 participants without missing data

*Three or more diagnoses from minimum three different organs/organ systems

(cough, $p=0.020$; voice change, $p=0.034$; Table 1). This was also the case when comparing participants with normal swallowing capacity; men exhibited more aspiration signs (cough, $p=0.038$; voice change, $p=0.004$; Supplementary data, Table Appendix 1). However, no significant differences were found between genders in the subgroup of participants with abnormal swallowing capacity (cough, $p=0.154$; voice change, $p=0.071$; Supplementary data, Table Appendix 2). Cough was observed in $20(22 \%)$ and voice change in 9 $(10 \%)$ of the 91 men with normal swallowing capacity.

\section{Risk of undernutrition}

A risk of undernutrition was found in 91 of 390 (23\%) participants and was associated with swallowing dysfunction $(p=0.033$; Table 3$)$. The risk of undernutrition was significantly higher among women $(60 / 209,29 \%)$ than men $(31 / 181,17 \% ; p=0.007)$. However, we found no association between the risk of undernutrition and age $(p=0.563)$, Katz-ADL $(p=0.387)$, multimorbidity $(p=0.215)$, dysphagia risk conditions $(p=0.945)$, or mild cognitive impairment $(p=0.278)$.

\section{Logistic regression analysis}

Participants with abnormal swallowing capacity had a higher risk of undernutrition compared to those with normal swallowing capacity (OR 1.74; 95\% CI 1.04-2.92) (Table 4). The risk of undernutrition was almost twice as high for females as for males (OR 1.93; 95\% CI 1.18-3.25).

\section{Discussion}

This descriptive, cross-sectional study of older people with different diagnoses who were admitted to Swedish shortterm care shows that the prevalence of swallowing dysfunction and undernutrition are high; more than half of all participants had an abnormal swallowing capacity, with onethird showing signs of aspiration and almost one-fourth at risk of undernutrition. Furthermore, abnormal swallowing capacity was found to be a risk factor for undernutrition. Despite the high prevalence of swallowing dysfunction, no routine screening is available for dysphagia among older people admitted to short-term care in Sweden [18]. The present study shows the necessity of developing such routines. To accomplish this, caregivers in short-term care in general need more resources and education to detect and manage dysphagia and risk of undernutrition.

The prevalence of dysphagia among older people varies greatly in the literature, from $11 \%$ in independently living older people to $80 \%$ among patients with neurodegenerative diseases or stroke [3]. The wide range is explained by both 
differences in settings and populations, and by the method of assessment, which is sometimes performed subjectively with questionnaires or objectively with clinical or instrumental assessments. In the present study, the prevalence of swallowing dysfunction was $63 \%$. This result may be explained by the diversity of the residents in the short-term care context and the use of a WST to detect swallowing dysfunction.

In older people, dysphagia is mostly reported to be associated with stroke and neurodegenerative diseases, or with aging itself $[4,8]$. In this study, swallowing dysfunction, including both signs of aspiration and swallowing capacity, was associated with increased age, whereas swallowing capacity alone was not. Anticipated changes in swallowing due to aging include a higher frequency of penetration (i.e., bolus entering the larynx but not proceeding below the true vocal folds) in healthy individuals 50 years of age and older [19]. However, the frequency of aspiration is not increased by age $[20,21]$. Another factor that may contribute to dysphagia, affecting swallowing safety and efficacy, is polymedication resulting in, e.g., declined saliva production, swallowing response or muscular relaxation [22, 23].

In the present study, swallowing dysfunction was also associated with general functional disability. This finding is in line with previous studies in which a reduction of independence in daily living was associated with dysphagia [9, 24]. The risk is that subjective symptoms of dysphagia, if associated with objectively measured swallowing dysfunction, leads to undernutrition that deteriorates functional capacity, which can lead to further deterioration of the swallowing dysfunction and negatively impact health and quality of life.

Earlier studies of healthy people under the age of 75 years showed that swallowing capacity is normally lower in women (mean $12.3 \mathrm{~mL} / \mathrm{s}$ ) than in men (mean $18.7 \mathrm{~mL} / \mathrm{s}$ ), but not less than $10 \mathrm{~mL} / \mathrm{s}$ [5, 25]. In the present study, abnormal swallowing capacity was significantly more common in women than in men, but men with normal swallowing capacity exhibited significantly more aspiration signs than women with normal swallowing capacity. People with swallowing dysfunction have been reported to compensate by reducing bolus size to prevent aspiration, which as a consequence reduces the swallowing capacity [26]. Therefore, we hypothesize that the women in this study have compensated or adapted to their dysfunction to a greater extent than the men by slowing down the speed of swallowing to avoid aspiration, whereas men more often continued to drink with normal capacity, with aspiration as a result. However, this hypothesis cannot be proved without an instrumental evaluation of swallowing with, e.g., videofluoroscopy or videoendoscopy. The difference between gender emphasizes the importance of not only assessing swallowing capacity, but also observing clinical signs of aspiration when screening for swallowing dysfunction.
Malnutrition is a common health problem among older people in various care facilities [9, 11, 27-29]. However, to the best of our knowledge, no study of nutritional status or dysphagia has been performed in the context of shortterm care. One-fourth of the participants in the present study were at risk of undernutrition, and the risk was associated with functional impairment and female gender. Furthermore, abnormal swallowing capacity was found to be a risk factor for undernutrition. Previous studies of older people in other settings have shown similar results. Among hospitalized older patients, dysphagia has been shown to be an independent risk factor for malnutrition [9]. In a geriatric outpatient clinic, $44 \%$ had poor nutritional status and malnutrition risk correlated with other geriatric syndromes [29].

A limitation of the study is that the results represent less than half of the population in short-term care due to a high rate of participant did not reach the inclusion criteria. Thus, the prevalence of swallowing dysfunction and risk of undernutrition may be underestimated, as the greater proportion were excluded due to insufficient cognitive capacity, and both dysphagia and malnutrition are known to be highly prevalent among people with dementia [3]. However, we found a tendency that participants with mild cognitive impairment had lower association with abnormal swallowing capacity compared to those with normal cognition. The inclusion criteria on sufficient cognitive capacity were based on medical records and subjective judgments by the nurse in charge. More objective would have been if it had been feasible to include a standardized evaluation, e.g., Mini-Mental State Examination [30], to the study protocol. However, it was not possible to add to the already extensive study protocol. To keep the protocol feasible for a study in a nonhospital context, no subjective measurement of swallowing dysfunction was performed.

The strengths of this study are that the screening tools were easy to use in a non-hospital context, and they are sensitive in identifying people at risk for swallowing dysfunction and undernutrition. This is important for future possible implementation of more systematic screening for swallowing dysfunction and risk of undernutrition in the short-term care context.

\section{Conclusion}

This study found high prevalence of swallowing dysfunction and risk of undernutrition among older people admitted to short-term care. Women exhibit poorer swallowing capacity than men, but men have clinical signs of aspiration more frequently than women. These results highlight the need for systematic, routine screening, feasible treatment to improve swallowing function, and strategies for safe food intake to 
improve health and prevent severe complications among older people in short-term care.

Acknowledgements We wish to thank Lena Olai, Eva Carlsson, Katri Ståhlnacke, and Mona Persenius from the SOFIA research group for their contributions and support.

\section{Compliance with ethical standards}

Conflict of interest The authors declare no conflict of interest.

Ethical approval This study was approved by the Uppsala Regional Ethics Review Board, Sweden (Dnr 2013/100), and was conducted in accordance with the Helsinki declaration (2013).

Informed consent All participants were included after providing written informed consent.

Open Access This article is distributed under the terms of the Creative Commons Attribution 4.0 International License (http://creativeco mmons.org/licenses/by/4.0/), which permits unrestricted use, distribution, and reproduction in any medium, provided you give appropriate credit to the original author(s) and the source, provide a link to the Creative Commons license, and indicate if changes were made.

\section{References}

1. Reynolds EW, Vice FL, Gewolb IH (2009) Variability of swallow-associated sounds in adults and infants. Dysphagia 24:13-19. https://doi.org/10.1007/s00455-008-9160-5

2. White JV, Guenter P, Jensen G et al (2012) Consensus statement of the Academy of Nutrition and Dietetics/American Society for Parenteral and Enteral Nutrition: characteristics recommended for the identification and documentation of adult malnutrition (undernutrition). J Acad Nutr Diet 112:730-738. https://doi. org/10.1016/j.jand.2012.03.012

3. Baijens LW, Clave P, Cras P et al (2016) European Society for Swallowing Disorders-European Union Geriatric Medicine Society white paper: oropharyngeal dysphagia as a geriatric syndrome. Clin Interv Aging 11:1403-1428. https://doi.org/10.2147/ CIA.S107750

4. Ortega O, Martin A, Clave P (2017) Diagnosis and management of oropharyngeal dysphagia among older persons, state of the art. J Am Med Dir Assoc 18:576-582. https://doi.org/10.1016/j.jamda 2017.02.015

5. Nathadwarawala KM, Nicklin J, Wiles CM (1992) A timed test of swallowing capacity for neurological patients. J Neurol Neurosurg Psychiatry 55:822-825

6. Wu MC, Chang YC, Wang TG et al (2004) Evaluating swallowing dysfunction using a $100-\mathrm{ml}$ water swallowing test. Dysphagia 19:43-47. https://doi.org/10.1007/s00455-003-0030-x

7. Logemann J (1998) Evaluation and treatment of swallowing disorders, 2nd edn. PRO-ED, Austin

8. Ney DM, Weiss JM, Kind AJ et al (2009) Senescent swallowing: impact, strategies, and interventions. Nutr Clin Pract 24:395-413. https://doi.org/10.1177/0884533609332005

9. Carrion S, Cabre M, Monteis R et al (2015) Oropharyngeal dysphagia is a prevalent risk factor for malnutrition in a cohort of older patients admitted with an acute disease to a general hospital. Clin Nutr 34:436-442. https://doi.org/10.1016/j.clnu.2014.04.014

10. National Alliance for Infusion $T$, the American Society for P, Enteral Nutrition Public Policy C, Board of D (2010)
Disease-related malnutrition and enteral nutrition therapy: a significant problem with a cost-effective solution. Nutr Clin Pract 25:548-554. https://doi.org/10.1177/0884533610378524

11. Kaiser MJ, Bauer JM, Ramsch C et al (2010) Frequency of malnutrition in older adults: a multinational perspective using the mini nutritional assessment. J Am Geriatr Soc 58:1734-1738. https:// doi.org/10.1111/j.1532-5415.2010.03016.x

12. Welfare NBoHa (2015) Åtgärdsförslag för att utveckla vården och omsorgen för de mest sjuka äldre. Primärvård och korttidsboende-vad behöver förändras? (Proposed measures to develop health and social care for the frailest elderly. Primary care and short-term care- what needs to be changed?). National Board of Health and Welfare in Sweden

13. Hagglund P, Olai L, Stahlnacke K et al (2017) Study protocol for the SOFIA project: swallowing function, oral health, and food intake in old age: a descriptive study with a cluster randomized trial. BMC Geriatr 17:78. https://doi.org/10.1186/s1287 7-017-0466-8

14. Brorsson B, Asberg KH (1984) Katz index of independence in ADL. Reliability and validity in short-term care. Scand J Rehabil Med 16:125-132

15. Katz S, Ford AB, Moskowitz RW et al (1963) Studies of illness in the aged. The index of Adl: a standardized measure of biological and psychosocial function. JAMA 185:914-919

16. Wallace M, Shelkey M, Hartford Institute for Geriatric N (2007) Katz index of independence in activities of daily living (ADL). Urol Nurs 27:93-94

17. Vallen C, Hagell P, Westergren A (2011) Validity and user-friendliness of the minimal eating observation and nutrition form-version II (MEONF- II) for undernutrition risk screening. Food Nutr Res. https://doi.org/10.3402/fnr.v55i0.5801

18. Edvinsson J, Rahm M, Trinks A et al (2015) Senior alert: a quality registry to support a standardized, structured, and systematic preventive care process for older adults. Qual Manag Health Care 24:96-101. https://doi.org/10.1097/QMH.0000000000000058

19. Daggett A, Logemann J, Rademaker A et al (2006) Laryngeal penetration during deglutition in normal subjects of various ages. Dysphagia 21:270-274. https://doi.org/10.1007/s0045 5-006-9051-6

20. Marik PE, Kaplan D (2003) Aspiration pneumonia and dysphagia in the elderly. Chest 124:328-336

21. Almirall J, Rofes L, Serra-Prat M et al (2013) Oropharyngeal dysphagia is a risk factor for community-acquired pneumonia in the elderly. Eur Respir J 41:923-928. https://doi.org/10.1183/09031 936.00019012

22. Stoschus B, Allescher HD (1993) Drug-induced dysphagia. Dysphagia 8:154-159

23. Sokoloff LG, Pavlakovic R (1997) Neuroleptic-induced dysphagia. Dysphagia 12:177-179. https://doi.org/10.1007/PL00009533

24. Inouye SK, Studenski S, Tinetti ME et al (2007) Geriatric syndromes: clinical, research, and policy implications of a core geriatric concept. J Am Geriatr Soc 55:780-791. https://doi.org/10.1 111/j.1532-5415.2007.01156.x

25. Hughes TA, Wiles CM (1996) Clinical measurement of swallowing in health and in neurogenic dysphagia. QJM 89:109-116

26. Buchholz DW, Bosma JF, Donner MW (1985) Adaptation, compensation, and decompensation of the pharyngeal swallow. Gastrointest Radiol 10:235-239

27. Saletti A, Lindgren EY, Johansson L et al (2000) Nutritional status according to mini nutritional assessment in an institutionalized elderly population in Sweden. Gerontology 46:139-145. https:// doi.org/10.1159/000022149

28. Suominen M, Muurinen S, Routasalo P et al (2005) Malnutrition and associated factors among aged residents in all nursing homes in Helsinki. Eur J Clin Nutr 59:578-583. https://doi.org/10.1038/ sj.ejcn.1602111 
29. Saka B, Kaya O, Ozturk GB et al (2010) Malnutrition in the elderly and its relationship with other geriatric syndromes. Clin Nutr 29:745-748. https://doi.org/10.1016/j.clnu.2010.04.006
30. Folstein MF, Folstein SE, McHugh PR (1975) "Mini-mental state". A practical method for grading the cognitive state of patients for the clinician. J Psychiatr Res 12:189-198 